ZAMM · Z, Angew. Math, u. Mech. 64 (1984) 7, 269-278

R. H. J. GMmith / C. R. TraAs

\title{
Optimization of Nordsieck's Method for the Numerical Integration of Ordinary Differential Equations
}

\begin{abstract}
Stabilität und Genauigkeit der Nordsieck-Methode können dadurch verbessert werden, daß die Anfangspositionen der parasitären Nullstellen in die komplexe Ebene verlegt werden. Dann werden optimale Anfangspositionen mit einer minimalen unteren Grenze für das Intervall der absoluten Stabilität und einem minimalen Koeffizienten im Abbruchfehler bestimmt. Schließlich werden mehrere Eigenschaften dieser verbesserten Methoden untersucht, zum Beispiel das Verhalten der höheren Ableitungen, die Aquivalenz mit Mehrschrittverfahren und die Dämpfung von Störungen.

Stability and accuracy of Nordsieck's integration method can be improved by choosing the zero-positions of the extraneous roots of the characteristic equation in a suitable way. Optimum zero-positions have been found by minimizing the lower bound of the interval of absolute stability and the coefficient of the truncation error. Various properties of the improved methods have been analysed, such as the behaviour of the high-order terms, the equivalence with multistep methods and the damping of perturbations.
\end{abstract}

Устойчивость и точность метода Нордзика монно улучшить при помони перевода нулевого положения посторониых корней в комплексную плоскость. Определяются оптимальные нулевые положения с минимальной нижней гранью для интервала абсолютной устойчивости и с минимальным коэффициентом в ошибке отбрасывания. Кроме того исследуются некоторые свойства этих улучшенных методов - па пример поведение производных выспего порядка, эвивалентность с многопаговыми методами и затухание возмущения.

\section{Introduetion}

NoRDSIEck introduced in his paper [12] a numerical integration method for a system of first-order ordinary differential equations

$$
y^{\prime}(x)=f(x, y(x)), \quad y\left(x_{0}\right)=y_{0}, \quad x \in\left[x_{0}, x_{n}\right]
$$

His method is essentially a one-step predictor-corrector method, equivalent to the classial Adams-Moulton multistep method. However, NonDsizck's formulation facilitates considerably the problems of stepsize change during the integration, and of starting the integration process.

It is known that any multistep integration method has a NoRDsIEck equivalent. A one-to-one correspondence exists between the values of the free parameters in the NorDsieck formulation and the multistep processes. In this article we describe how the Nordsieck parameters must be chosen in order to obtain integration methods with improved properties relative to the original Nordsieck method, as far as truncation error and size of stability region are concerned, and we give the equivalent multistep method. We also analyse the damping characteristics of these modified methods with respect to perturbations.

Most of the complicated calculations in our paper were carried out with the formula-manipulation system REDUCE [7].

\section{Nordsieck's method}

In this paper we restrict our analysis to the Nordsieck method based on a fifth-degree polynomial approximation, $m=5$. Both stability and accuracy of this method are satisfactory in a majority of practical applications.

The following working equations define the one-step method with a stepsize $h$ :

$$
\begin{aligned}
& y^{[\mathrm{p}]}=y(x)+h \cdot\{f(x, y(x))+ \\
& a(x)+b(x)+c(x)+d(x)\}, \\
& f^{[\mathrm{p}]}=f(x, y(x))+ \\
& +2 a(x)+3 b(x)+4 \cdot c(x)+5 \cdot d(x), \\
& y(x+h)=y^{[\mathrm{p}]} \quad+h \cdot Y \cdot\left[f(x+h, y(x+h))-f^{[\mathrm{p}]}\right] \text {, } \\
& a(x+h)=a(x)+3 b(x)+6 \cdot c(x)+10 \cdot d(x)+A \cdot\left[f(x+h, y(x+h))-f^{[\mathrm{p}]}\right] \text {, } \\
& b(x+h)=\quad b(x)+4 \cdot c(x)+10 \cdot d(x)+B \cdot\left[f(x+h, y(x+h))-f^{[p]}\right], \\
& c(x+h)=\quad c(x)+5 \cdot d(x)+C \cdot\left[f(x+h, y(x+h))-f^{[\mathrm{p}]}\right], \\
& d(x+h)=\quad d(x)+D \cdot\left[f(x+h, y(x+h))-f^{[\mathrm{p}]}\right] .
\end{aligned}
$$

The predicted values $y^{[\mathrm{p}]}$ and $f^{[\mathrm{p}]}$ represent initial estimates for $y(x+h)$ and $f(x+h, y(x+h))$.

The Nordsieck vector

$$
[y(x), h \cdot f(x, y(x)), h \cdot a(x), h \cdot b(x), h \cdot c(x), h \cdot d(x)]^{\top}
$$

approximates high-order derivatives of the solution $y(x)$ in Taylor form

$$
\left[y(x), h \cdot y^{\prime}(x), \frac{h^{2}}{2 !} \cdot y^{\prime \prime}(x), \frac{h^{3}}{3 !} \cdot y^{\prime \prime \prime}(x), \frac{h^{4}}{4 !} \cdot y^{\prime \prime \prime \prime}(x), \frac{h^{5}}{5 !} \cdot y^{\prime \prime \prime \prime \prime}(x)\right]^{\top}
$$


The functions $a(x), b(x), c(x)$ and $d(x)$ are the high-order terms of the integration method. The presence of the derivatives up to the fifth-order guarantees the existence of a unique fifth-degree polynomial, approximating the solution of the differential equation.

NoRDsieck's predictor can be seen as the result of a multiplication of the vector (3) by an uppertriangular Pascal matrix. The corrector term $\left[f(x+h, y(x+h))-f^{[p]}(x)\right]$ is used to improve the predicted values. values

The requirement of optimum stability (all 4 extraneous roots in the origin for $h=0$ ) results in the coefficient

$$
A=25 / 24, \quad B=35 / 72, \quad C=5 / 48, \quad D=1 / 120
$$

The remaining coefficient $Y$ minimizes the truncation error:

$$
Y=95 / 288 \text {. }
$$

The unknown $y(x+h)$ is solved from the implicit equation $(2 \mathrm{c})$, using iteration with only 2 function-evaluations $f\left(x_{i}, y_{i}\right)$ :

$$
\left.\begin{array}{ll}
y^{(1)}(x+h)=y^{[p]}, & f^{(1)}(x+h)=f\left(x+h, y^{(1)}(x+h)\right), \\
y^{(2)}(x+h)=y^{[p]}+h \cdot Y \cdot\left[f^{(1)}(x+h)-f^{[p]}\right], & f^{(2)}(x+h)=f\left(x+h, y^{(2)}(x+h)\right), \\
y^{(3)}(x+h)=y^{[p]}+h \cdot Y \cdot\left[f^{(2)}(x+h)-f^{[p]}\right] . &
\end{array}\right\}
$$

\section{Stability}

The characteristic equation $\psi(\lambda, p)=0$, associated with the system $(2)$, is a complex fifth-degree polynomial in $\lambda$ :

$$
\begin{aligned}
\psi(\lambda, p)= & {\left[\lambda^{5}+\lambda^{4} \cdot(2 A+3 B+4 C+5 D-5)+\lambda^{3} \cdot(-8 A-6 B+8 C+50 D+10)+\right.} \\
& +\lambda^{2} \cdot(12 A-24 C-10)+\lambda \cdot(-8 A+6 B+8 C-50 D+5)+(2 A-3 B+4 C-5 D \\
& -1)]- \\
& -p \cdot\left[\lambda^{5} \cdot Y+\lambda^{4} \cdot(1+A+B+C+D-5 Y)+\lambda^{3} \cdot(-4-2 A+2 B+10 C+26 D+\right. \\
& +10 Y)+ \\
& +\lambda^{2} \cdot(-6 B+66 D-10 Y+6)+\lambda \cdot(2 A+2 B-10 C+26 D+5 Y-4)+ \\
& +(1-A+B-C+D-Y)] .
\end{aligned}
$$

The stability parameter $p$ is complex and depends on the stepsize $h$ and the Jacobian matrix $J$ of the system of differential equations

$$
J=\left[\frac{\partial f}{\partial y}\right] .
$$

The roots $\lambda$ of the characteristic equation (or equivalently, the eigenvalues of the stability matrix $M$, see [12], p. 28) are classified as follows:

$$
\left.\begin{array}{lll}
\text { "principal" root: } & \lambda=\lambda_{0}=1 \text { for } p=0, \\
\text { "extraneous" roots: } & \lambda=\lambda_{i}=z_{i} \text { for } p=0, \quad i=1,2,3,4,
\end{array}\right\}
$$

( $z_{i}$ is real, or it occurs in complex-conjugate pairs).

We have used various techniques to investigate the stability of NordsIEck methods:

1. Boundary Locus Method (as a direct consequence of $\psi(\lambda, p)$ linear in $p$ ).

2. Theorem of RovTH-HunwITz.

3. Numerical solution of the characteristic equation.

Definition: The region $G$ which is such that, for every $p \in G$, the roots of $\psi(\lambda, p)=0$ satisfy

$|\lambda(p)| \leqq 1$ when $\lambda(p)$ is a simple root, $\quad|\lambda(p)|<1$ when $\lambda(p)$ is a multiple root,

is called the region of absolute stability.

Figure 1 shows the region of absolnte stability for NoRDsIEck's choice of coefficients (5), (6), corresponding to 4 extraneous zero-positions in the origin: $z_{i}=0, i=1,2,3,4$.

The integration method is absolutely stable, if for all eigenvalues $\xi$ of the Jacobian matrix $J$, the stability parameter $p=h \cdot \xi$ lies inside the region $G$ of absolute stability.

The interval of absolute stability $\left[p_{\min }, 0\right]$ is the intersection of $G$ with the real axis. The value $p=p_{\min }$ represents an absolutely stable situation, with one simple extraneous root $\lambda=-1$. The lower bound $p_{\min }$ depends on the coefficient values

$$
p_{\min }=\frac{4 \cdot(1-A+C)}{B-2 D+4 Y-2}
$$

For the optimum stable method (5) applies:

$$
p_{\min }=-45 / 38 \approx-1.18421 \text {. }
$$




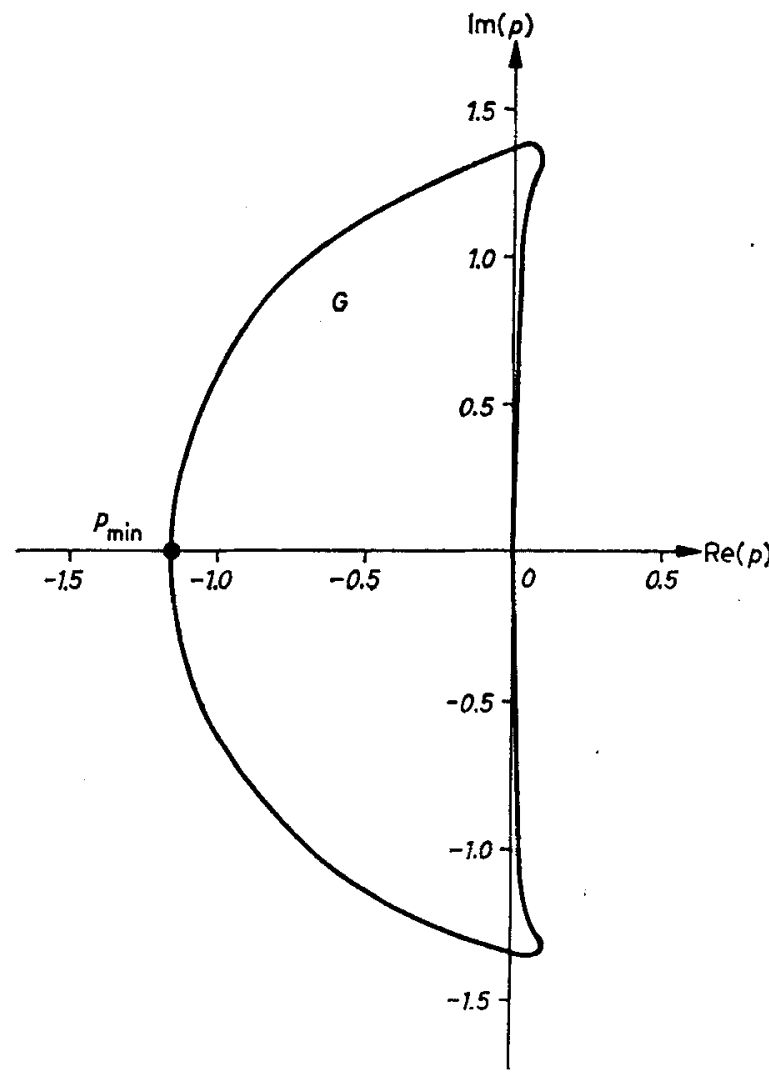

Fig. 1. Region of absolute stability for the original Nordsieck method, $A=$ $=25 / 24, B=35 / 72, C=5 / 48, D=1 / 120, Y=95 / 288, \quad p_{\min }=-45 / 38$

Definition: The region $R$ which is such that, for every $p \in R$, the roots of $\psi(\lambda, p)=0$ satisfy

$$
\left|\lambda_{i}(p)\right| \leqq\left|\lambda_{0}(p)\right|, \quad i=1,2,3,4,
$$

is called the region of relative stability.

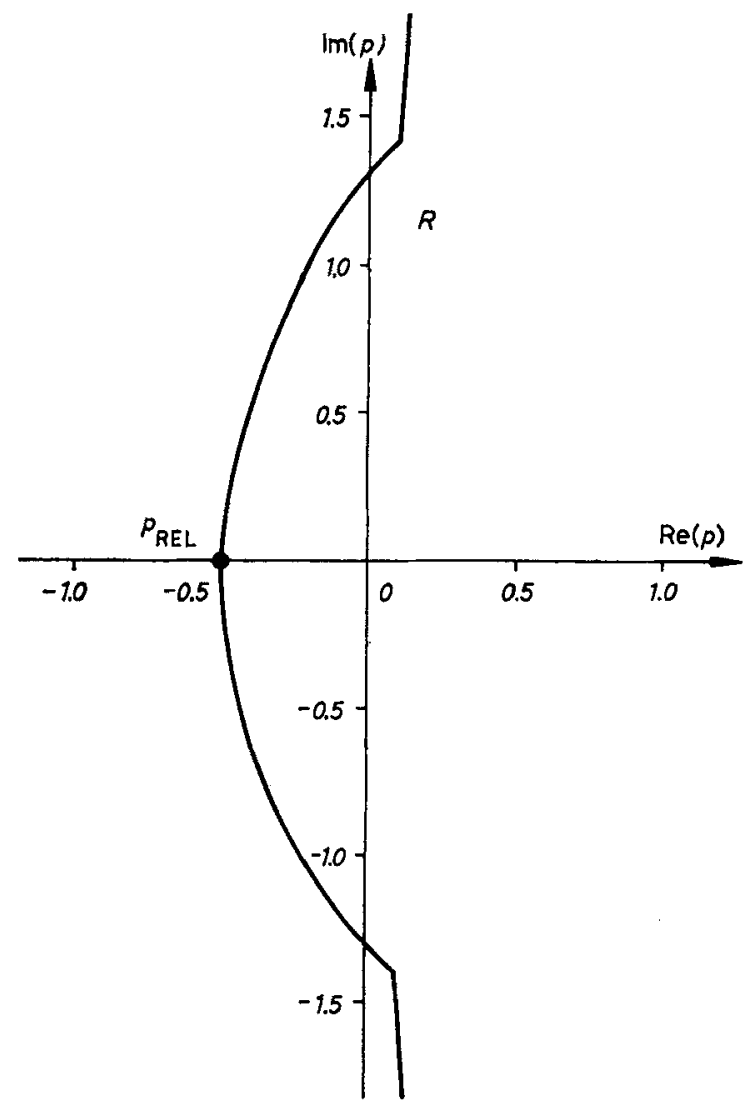

Fig. 2. Region of relative stability for the original Nordsieck method, $A=25 / 24$ $B=35 / 72, C=5 / 48, D=1 / 120, Y=95 / 288, p_{\text {rel }} \simeq-0.494$

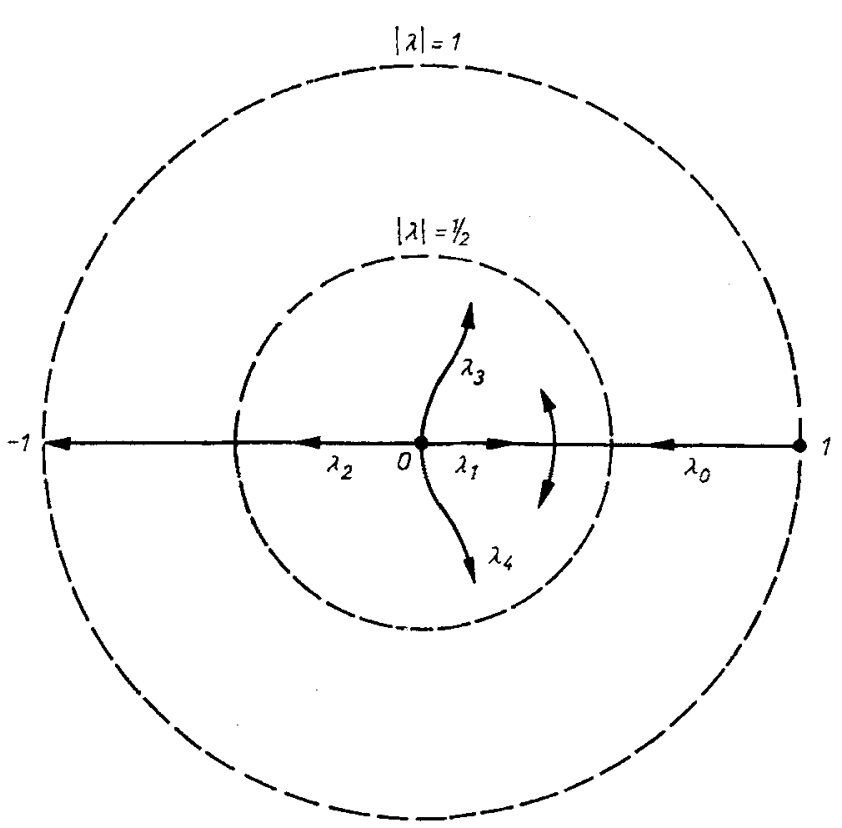

Fig. 3. Roots moving away from the zero-positions, $p$ negative, real, $z_{1}=z_{2}=$ 
In figure 2 the region of relative stability in the complex $p$-plane is given for the original method (5), (6).

The roots $\lambda(p)$ strongly depend on the value of the stability parameter $p$. Figure 3 indicates how these roots move away from their zero-positions in the origin, for negative values of $p$. Instability for $p<p_{\min }$ is caused by a negative extraneous root, intersecting the unit circle at $\lambda=-1$. After collision $(p \approx-0.93)$ the positive extraneous root together with the principal root form a conjugate-complex pair. Compare the results of BRowN, RILEY and Benn ETT [1] for the Adams-Moulton method.

\section{Accuracy}

The principal root $\lambda_{0}$ and the truncation error $T$ are related by the formula

$$
\lambda_{0}(p)=\mathrm{e}^{p}+T(p)
$$

Substituting a Taylor series expansion around $p=0$, for $\lambda_{0}(p)$ in the characteristic equation and solving $T$ from (15) give

$$
T(h)=\frac{30 Y+5 A-C-15}{5 \cdot D} \cdot \frac{h^{6}}{6 !} \cdot \frac{\mathrm{d}^{6} y}{\mathrm{~d} x^{6}}+H \cdot \frac{h^{7}}{7 !} \cdot \frac{\mathrm{d}^{7} y}{\mathrm{~d} x^{7}}+O\left(h^{8}\right) .
$$

The coefficient $Y$ can be chosen to minimize the truncation error (eliminating $h^{6}$ from (16)):

$$
\left.\begin{array}{l}
Y(A, B, C, D)=1 / 2-A / 6+\mathrm{C} / 30, \quad T(h)=H \cdot \frac{h^{7}}{7 !} \cdot \frac{\mathrm{d}^{7} y}{\mathrm{~d} x^{7}}+O\left(h^{8}\right), \\
H=\frac{35-35 A+14 B+7 \mathrm{C}-10 D}{10 \cdot D} .
\end{array}\right\}
$$

With this choice $Y$ depends entirely on the values of $A, B, C$ and $D$. Therefore, the optimization of the integration method can be accomplished by varying the coefficients $A$ through $D$ only. Putting all extraneous zero-positions in the origin yields for the coefficient of the truncation error

$$
H=863 / 12 \approx 71.91667 \text {. }
$$

Nordsieck $m=\mathbf{5}$ is a sixth-order method, capable of exact integration of ordinary differential equations, with polynomial solutions up to and including degree 6. DAHLQUIST's theorem ([5], 2, p. 160) states that no values of $A$, $B, C$ and $D$ exist such that a zero-stable seventh-order method $\left(T \sim h^{8}\right)$ arises.

Neglecting roundoff errors, the global discretisation error $e(x)$ is

$$
e(x) \approx \frac{H}{120 \cdot D} \cdot \frac{h^{6}}{7 !} \cdot\left(x-x_{0}\right) \cdot \frac{\mathrm{d}^{7} y}{\mathrm{~d} x^{7}}+O\left(h^{7}\right) .
$$

The zero-positions of the extraneous roots obey the equation

$$
\left(1-z_{1}\right)\left(1-z_{2}\right)\left(1-z_{3}\right)\left(1-z_{4}\right)=120 \cdot D
$$

and therefore establish the correspondence with the results of FEHLBERG [3] and HULL [9]. These authors recommended extraneous zero-positions, with maximum distance to the principal zero-position $\lambda_{0}=1$, minimizing the coefficient $H /(120 \cdot D)$ of the global discretisation error.

\section{Shifting the extraneous zero-positions}

We try to improve stability and accuracy of the $m=5$ method by shifting the zero-positions of the extraneous roots, while preserving the structure of NorDsIEck's method. The idea is, on the one side, to slow down the shift of the extraneous roots towards the unit circle when increasing the value of $|p|$ in order to enlarge the region of absolute stability, and on the other side to decrease the value of the truncation coefficient $H$ in order to obtain a more accurate integration method.

Changing the zero-positions $z_{i}$ affects the coefficient values $A, B, C, D, Y$ of the corrector, without violating the lower-order predictor terms (conserving the order of accuracy). The need for consistency forbids shifting the principal zero-position.

Under the condition of real values for the Nordsieck coefficients there exist three families of extraneous zeropositions:

1. 4 real extraneous roots:

$$
z_{1}=z_{11}, \quad z_{2}=z_{21}, \quad z_{3}=z_{31}, \quad z_{4}=z_{41} ;
$$

2. 2 real and 1 pair of conjugate-complex extraneous roots :

$$
z_{1}=z_{11}, \quad z_{2}=z_{21}, \quad z_{3}=z_{31}+i \cdot z_{32}, \quad z_{4}=\bar{z}_{3}
$$

3. 2 pairs of conjugate-complex extraneous roots:

$$
z_{1}=z_{11}+i \cdot z_{12}, \quad z_{2}=\bar{z}_{1}, \quad z_{3}=z_{31}+i \cdot z_{32}, \quad z_{4}=\bar{z}_{3} .
$$


The relationship between NoRDsIEck's coefficients and the extraneous zero-positions is determined by the characteristic equation (8), (22) for $p=0$ :

$$
\begin{aligned}
& \psi(\lambda, p=0)=(\lambda-1) \cdot\left(\lambda-z_{1}\right) \cdot\left(\lambda-z_{2}\right) \cdot\left(\lambda-z_{3}\right) \cdot\left(\lambda-z_{4}\right), \\
& T_{1}=\left(z_{1}-1\right)+\left(z_{2}-1\right)+\left(z_{3}-1\right)+\left(z_{4}-1\right) \text {, } \\
& T_{2}=\left(z_{1}-1\right) \cdot\left(z_{2}-1\right)+\left(z_{1}-1\right) \cdot\left(z_{3}-1\right)+\left(z_{1}-1\right) \cdot\left(z_{4}-1\right)+\left(z_{2}-1\right) \cdot\left(z_{3}-1\right)+ \\
& +\left(z_{2}-1\right) \cdot\left(z_{4}-1\right)+\left(z_{3}-1\right) \cdot\left(z_{4}-1\right) \text {, } \\
& T_{3}=\left(z_{1}-1\right) \cdot\left(z_{2}-1\right) \cdot\left(z_{3}-1\right)+\left(z_{1}-1\right) \cdot\left(z_{2}-1\right) \cdot\left(z_{4}-1\right)+\left(z_{1}-1\right) \cdot\left(z_{3}-1\right) \cdot\left(z_{4}-1\right)+ \\
& +\left(z_{2}-1\right) \cdot\left(z_{3}-1\right) \cdot\left(z_{4}-1\right) \text {, } \\
& T_{4}^{\prime}=\left(z_{1}-1\right) \cdot\left(z_{2}-1\right) \cdot\left(z_{3}-1\right) \cdot\left(z_{4}-1\right) \text {; } \\
& A=-T_{1} / 2-T_{2} / 4-T_{3} / 6-T_{4} / 8 \text {, } \\
& B==\quad T_{2} / 6+T_{3} / 6+11 \cdot T_{4} / 72, \\
& C=\quad-T_{3}^{\prime} / 24-T_{4} / 16 \text {, } \\
& D=\quad T_{4} / 120 \text {, } \\
& Y=Y(A, B, C, D)=1 / 2+T_{1} / 12+T_{2} / 24+19 \cdot T_{3} / 720+3 \cdot T_{4} / 160 .
\end{aligned}
$$

The original coefficient values (5), (6) are recovered when substituting $z_{1}=z_{2}=z_{3}=z_{4}=0$ in the formulas (23). For given coefficients in the working equations the extraneous zero-positions can be found as the roots $\left\{1, z_{1}, z_{2}\right.$, $\left.z_{3}, z_{4}\right\}$ of the characteristic equation $\psi(\lambda, p=0$ ) (see STETTER [16]). The relations (22), (23) form the unique correspondence between roots and coefficients. Any feasible Nordsieck method is thus precisely determined by the 4 extraneous zero-positions. As a consequence of zero-stability $(p=0)$ the roots must always satisfy

$$
\left.\begin{array}{l}
\left|z_{i}\right| \leqq 1, \quad z_{i} \neq 1, \quad z_{i} \text { a simple root }, \\
\left|z_{i}\right|<1, \quad z_{i} \text { a multiple root } .
\end{array}\right\}
$$

\section{Optimization}

Stability and accuracy of the integration method depend completely on the zero-positions, chosen from one of the families (21). The objective of the optimization is to minimize the coefficient of the truncation error

$$
H=\frac{35-35 A+14 B+7 C-10 D}{10 D}
$$

and to maximize the region of absolute stability (to minimize the lower bound of the interval of absolute stability)

$$
p_{\text {min }}=\frac{-60 \cdot(1-A+C)}{10 A-15 B-2 C+30 D} .
$$

During the optimization also the influence of the extraneous zero-positions on the size and the form of the absolute stability region in the complex $p$-plane is considered.

The following optimization problems have been solved:

problem 1: minimize the coefficient $H$ of the truncation error, while preserving at least the stability of NorDsIECK's original method:

$$
\left.\begin{array}{ll}
\text { minimize } & H\left(z_{1}, z_{2}, z_{3}, z_{4}\right), \\
\text { subject to: } & p_{\text {min }}\left(z_{1}, z_{2}, z_{3}, z_{4}\right) \leqq-45 / 38 ;
\end{array}\right\}
$$

problem 2: minimize the lower bound $p_{\text {min }}$ of the absolute stability interval, preserving at least the accuracy of NorDsreck's original method $(5),(6)$ :

$\left.\begin{array}{ll}\text { minimize } & p_{\min }\left(z_{1}, z_{2}, z_{3}, z_{4}\right) \\ \text { subject to: } & H\left(z_{1}, z_{2}, z_{3}, z_{4}\right) \leqq 863 / 12 ;\end{array}\right\}$

problem 3 : as a compromise, improve both the accuracy and the stability by the same maximum fraction $\alpha$ :

$$
\left.\begin{array}{ll}
\text { maximize } & \alpha\left(z_{1}, z_{2}, z_{3}, z_{4}\right), \\
\text { subject to: } & \frac{863 / 12-H\left(z_{1}, z_{2}, z_{3}, z_{4}\right)}{863 / 12} \geqq \alpha, \quad \frac{-45 / 38-p_{\min }\left(z_{1}, z_{2}, z_{3}, z_{4}\right)}{45 / 38} \geqq \alpha .
\end{array}\right\}
$$

The need for sufficient error damping in Nordsieck methods restricts the class of possible optimum zero-positions to

$$
\left|z_{i}\right| \leqq 1 / 2, \quad i=1,2,3,4 \text {. }
$$

Zero-positions were found, using FORTRAN-routines for minimizing/maximizing a function, subject to a number of constraints. A combination of two pairs of conjugate-complex extraneous zero-positions (family 3 ) gave the best results for both stability and accuracy. The following optimum Nordsieck methods were found, satisfying condition (30):

$\left.\begin{array}{rl}\text { problem } 1: z_{1} & =-0.20379+i \cdot 0.45658, \quad z_{2}=\bar{z}_{1}, \quad z_{3}=-0.16313+i \cdot 0.47264, \quad z_{4}=\bar{z}_{3}, \\ H & =25.17272(<863 / 12), \quad p_{\min }=-1.18420(\approx-45 / 38)\end{array}\right\}$ 
problem $2: z_{1}=0.03973+i \cdot 0.49842, \quad z_{2}=\bar{z}_{1}, \quad z_{3}=0.08187+i \cdot 0.49325, \quad z_{4}=\bar{z}_{3}$

$$
\left.\begin{array}{rl}
H & =71.91626(\approx 863 / 12), \quad p_{\min }=-2.24754(<-45 / 38) ; \\
\text { problem } 3: z_{1} & =-0.07582+i \cdot 0.49421, \quad z_{2}=\vec{z}_{1}, \quad z_{3}=-0.05008+i \cdot 0.49747, \quad z_{4}=\bar{z}_{3},\{ \\
H & =40.95460, \quad p_{\min }=-1.69403, \quad \text { simultaneous improvement } \alpha=0.431
\end{array}\right\}
$$

(all zero-positions in 5 significant digits).

Formula-manipulation techniques have shown, for methods chosen from family 3

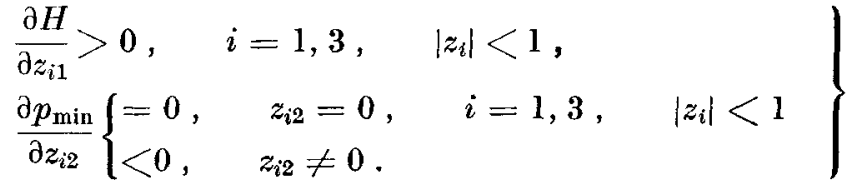

From these properties of the partial derivatives a global confirmation can be obtained regarding the optimality of the zero-positions found above.

Shifting zero-positions to the left half-plane (maximizing the distance to $\lambda_{0}=1$ ) improves the accuracy of the integration method (see also HULL [9]: Westward formulas). Increasing the imaginary parts $z_{i 2}$ enlarges the region of absolute stability. With increasing $|p|$ conjugate-complex extraneous roots move substantially slower, away from their zero-positions, than real extraneous roots.

Hull and Nwwbery [9] state that a multiplicity of the roots $z_{i}$ at their zero-positions increases the speed of their outward motion with growing $|p|$, if compared with the outward speed of simple roots. For this reason they recommend integration methods of the latter type, i.e. with simple roots. However, this effect only occurs for small values of $|p|$, and therefore it can not serve as an argument for assertions about absolute stability. Instead, the boundary locus method should be used to investigate the stability properties. For this reason our optimal methods (31), (32), (33), which were obtained along these lines, do not correspond with the West-Radial formulas proposed in [9] as a compromise between stability and accuracy.

\section{Behaviour of the high-order terms}

The functions $a(x), b(x), c(x), d(x)$ represent the "memory" of the integration process. Solving series expansions for these quantities from the working equations $(2)$ leads to

$$
\begin{aligned}
a(x)= & \frac{h}{2 !} \cdot y^{\prime \prime}(x)+\frac{-6+6 A-3 B+D}{2 D} \cdot \frac{h^{5}}{6 !} \cdot \frac{\mathrm{d}^{6} y}{\mathrm{~d} x^{6}}+ \\
& +\frac{21 B C-42 A C-35 C D+70 A D+42 C-105 D}{10 \cdot D^{2}} \cdot \frac{h^{6}}{7 !} \cdot \frac{\mathrm{d}^{7} y}{\mathrm{~d} x^{7}}+O\left(h^{7}\right) \\
b(x)= & \frac{h^{2}}{3 !} \cdot y^{\prime \prime \prime}(x)+\frac{-2 A+3 B-2 C}{D} \cdot \frac{h^{5}}{6 !} \cdot \frac{\mathrm{d}^{6} y}{\mathrm{~d} x^{6}}+ \\
& +\frac{28 C^{2}+35 B D-35 D^{2}-42 B C+28 A C-70 D}{10 \cdot D^{2}} \cdot \frac{h^{6}}{7 !} \cdot \frac{\mathrm{d}^{7} y}{\mathrm{~d} x^{7}}+O\left(h^{7}\right), \\
c(x)= & \frac{h^{3}}{4 !} \cdot y^{\prime \prime \prime \prime}(x)+\frac{-6 B+12 C-10 D}{4 D} \cdot \frac{h^{5}}{6 !} \cdot \frac{\mathrm{d}^{6} y}{\mathrm{~d} x^{6}}+ \\
& +\frac{-42 C^{2}+70 C D+21 B C-35 A D}{10 \cdot D^{2}} \cdot \frac{h^{6}}{7 !} \cdot \frac{\mathrm{d}^{7} y}{\mathrm{~d} x^{7}}+O\left(h^{7}\right), \\
d(x)= & \frac{h^{4}}{5 !} \cdot y^{\prime \prime \prime \prime \prime}(x)+\frac{15 D-6 C}{5 D} \cdot \frac{h^{5}}{6 !} \cdot \frac{\mathrm{d}^{6} y}{\mathrm{~d} x^{6}}+\frac{84 C^{2}-105 B D+175 D^{2}-210 C D}{50 \cdot D^{2}} \cdot \frac{h^{6}}{7 !} \cdot \frac{\mathrm{d}^{7} y}{\mathrm{~d} x^{7}}+O\left(h^{7}\right) .
\end{aligned}
$$

These high-order functions "lag" behind the current value of the integration variable $x$. The last of the equations (35) can be reformulated as

$$
d(x)=\frac{h^{4}}{5 !} \cdot \frac{\mathrm{d}^{5} y}{\mathrm{~d} x^{5}}\left(x-\frac{2 C-5 D}{10 \cdot D} \cdot h\right)+O\left(h^{6}\right)
$$

so that the lag in $d(x)$ is

$$
\operatorname{lag}[d(x)]=\frac{2 C-5 D}{10 \cdot D} \cdot h
$$

For the original Nordsieck method $(\mathbf{5}),(6)$

$$
\operatorname{lag}[d(x)]=2 \cdot h \text {. }
$$

The lag in $a(x), b(x), c(x)$ depends on the integration variable $x$ and cannot be expressed in formula form, free of $x$. NoRDSIECK stated in his paper

$$
\operatorname{lag}[a(x)]<\operatorname{lag}[b(x)]<\operatorname{lag}[c(x)]<\operatorname{lag}[d(x)] .
$$


The "lagging behind" is essentially a consequence of stabilizing the working equations. Coefficient values, derived directly from Taylor series,

$$
A=5 / 2, \quad B=10 / 3, \quad C=5 / 2, \quad D=1, \quad Y=1 / 6
$$

yield high-order functions with no lag. This method, however, is not zero-stable

$$
z_{1}=-0.043, \quad z_{2}=-0.431, \quad z_{3}=-2.322, \quad z_{4}=-23.204 .
$$
p. 31).

Formulas (35) determine an easy rule for adapting high-order terms, when changing the stepsize $h$ (see [12],

NORDSIECK's corrector expression satisfies

$$
\left[f(x+h)-f^{[\mathrm{p}]}(x)\right]=\frac{1}{D} \cdot \frac{h^{5}}{5 !} \cdot \frac{\mathrm{d}^{6} y}{\mathrm{~d} x^{6}}\left(x-\frac{C-\mathbf{5} D}{5 D} \cdot h\right)+O\left(h^{7}\right), \quad \text { lag }=\frac{C-\mathbf{5} D}{5 D} \cdot h .
$$

For the original method $z_{i}=0, i=1,2,3,4$, this lag amounts to

$$
\operatorname{lag}\left[f(x+h)-f^{[p]}(x)\right]=\frac{3}{2} \cdot h \text {. }
$$

\section{Equivalence with multistep methods}

NoRDsiEch's original one-step formula is equivalent with the classical 5-step implicit Adams-Moulton method (GEAR [4], Osborne [14]). This correspondence is based on an equivalent fifth-degree polynomial approximation for the solution of the differential equations. Instead of the vector (4) with higher derivatives in a single point $x$, ADAMs uses function values $y^{\prime}=f$ in $\mathbf{5}$ previous points:

$$
\left[y(x), h \cdot y^{\prime}(x), h \cdot y^{\prime}(x-h), h \cdot y^{\prime}(x-2 h), h \cdot y^{\prime}(x-3 h), h \cdot y^{\prime}(x-4 h)\right]^{\top} .
$$

Nordsieck methods, with shifted extraneous zero-positions, are equivalent with general linear implicit 5-step methods, not of Adams type:

$$
\begin{array}{ll}
y(x+h)+S_{1} \cdot y(x)+S_{2} \cdot y(x-h)+S_{3} \cdot y(x-2 h)+S_{4} \cdot y(x-3 h)+S_{5} \cdot y(x-4 h)= \\
=h \cdot\left[U_{0} \cdot f(x+h)+U_{1} \cdot f(x)+U_{2} \cdot f(x-h)+U_{3} \cdot f(x-2 h)+U_{4} \cdot f(x-3 h)+U_{5} \cdot f(x-4 h)\right], \\
S_{1}=2 A+3 B+4 C+5 D-5, & U_{0}=Y, \\
S_{2}=-8 A-6 B+8 C+50 D+10, & U_{1}=A+B+C+D-5 Y+1, \\
S_{3}=12 A-24 C-10, & U_{2}=-2 A+2 B+10 C+26 D+10 Y-4, \\
S_{4}=-8 A+6 B+8 C-50 D+5, & U_{3}=-6 B+66 D-10 Y+6, \\
S_{5}=2 A-3 B+4 C-5 D-1, & U_{4}=2 A+2 B-10 C+26 D+5 Y-4,
\end{array}
$$

(compare the coefficients of the characteristic equation (8)).

Only the choice of maximum zero-stability results in a method of Adams type

$$
\left.\begin{array}{l}
z_{1}=z_{2}=z_{2}=z_{4}=0 \Leftrightarrow S_{2}=S_{3}=S_{4}=S_{5}=0, \\
\exists i \in\{1,2,3,4\}: z_{i} \neq 0 \Leftrightarrow \exists i \in\{2, \mathbf{3}, 4,5\}: S_{i} \neq 0 .
\end{array}\right\}
$$

GRIGORLEFF [5] proved the existence of a bijection between the class of Nordsieck methods and the class of linear implicit multistep methods.

\section{Reduction of transients}

Transients during integration occur as a result of shocks, such as changing the interval size $h$, reversing the direction of the start, or a discontinuity in the right-hand side $f(x, y)$ of the differential equations. These disturbances cause oscillations in the high-order terms $a(x), b(x), c(x), d(x)$ and $\left[f(x+h)-f^{[p]}(x)\right]$.

The response of any stable Nordsieck method to a shock is a damping of the oscillations. In practical implementations the method must be given the opportunity to damp the oscillations sufficiently. For this reason the stepsize $h$ must be kept constant for a number of integration steps after a shock has occurred.

We will investigate the influence of the extraneous zero-positions on the transient reduction. OsBorNE [14] gives a formula for the error $\Delta u_{i+q}$ in the high-order terms, as a result of an initial disturbance $\Delta u_{i}$ in $x_{i}$ :

$$
u_{i}=\left[a\left(x_{i}\right), b\left(x_{i}\right), c\left(x_{i}\right), d\left(x_{i}\right)\right]^{\top}, \quad \Delta u_{i+q}=S^{q} \cdot \Delta u_{i}+L_{i, q}(f, S) \cdot \gamma
$$

where

$$
\begin{aligned}
& S=\left[\begin{array}{rrrr}
1-2 A & 3-3 A & 6-4 A & 10-5 A \\
-2 B & 1-3 B & 4-4 B & 10-5 B \\
-2 C & -3 C & 1-4 C & 5-5 C \\
-2 D & -3 D & -4 D & 1-5 D
\end{array}\right], \quad \gamma=[A, B, C, D]^{\top}, \\
& L_{i, q}(f, S)=\sum_{k=0}^{q-1}\left(f_{i+q-k}-f_{i+q-k-1}\right) \cdot S^{k}
\end{aligned}
$$


The reduction matrix $S$ is a part of the stability matrix $M$, substituting $p=0$ and omitting the first row and the first column of $M$. Then the eigenvalues of $S$ are precisely the extraneous zero-positions $z_{i}$ of the Nordsieck method.

The first part of equation (47): $\mathrm{S}^{q} \cdot \Delta u_{i}$ is an oscillating contribution to the error, due to the high-order terms. When all the extraneous zero-positions lie strictly inside the unit circle, this contribution can be made arbitrarily small for a sufficiently large number of steps $q$.

The second part: $L_{i, q}(f, S) \cdot \gamma$ contains, together with an oscillating part, also a persistent contribution to the error, which can only be reduced by decreasing the stepsize $h$. For trivial differential equations (right-hand side $f$ constant)

$$
L_{i, q}(f, S)=\mathbf{0} \text {, }
$$

and all shock-excited errors will disappear.

The error in the numerical solution $y$ caused by a shock, is smaller by one order $h$ compared to the error $\Delta u_{i}$ in the high-order terms.

Theorem: For Nordsieck's method $m=\mathbf{5}$

1. $S^{4}=0$ if and only if $z_{1}=z_{2}=z_{3}=z_{4}=0$ (optimum zero-stability);

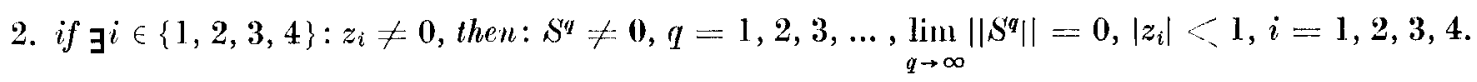

Proof: Using REDUCE formula-manipulation and the Cayley-Hamilton theorem, which states that the matrix $S$ satisfies its own characteristic equation $\psi(S)=\mathbf{0}$.

Only the original Nordsieck method $m=5$ eliminates $S^{q}$-oscillations in precisely 4 integration steps. 'This property is lost if at least one of the extraneous zero-positions $z_{i}$ is not in the origin. These methods need an infinite number of steps to damp the oscillations in the high-order terms completely. This infinite-interval transient reduction is not a serious disadvantage during practical numerical integration.

The asymptotic rate of the error damping is determined by

$$
\underset{i=1,2,3,4}{\operatorname{maximmm}}\left|z_{i}\right| \text {. }
$$

To safeguard the quality of the transient elimination, it should be recommended to place the extraneous zeropositions "not too far" from the origin in the complex $\lambda$-plane.

\section{Integration program}

'The algorithm for the numerical integration is practically identical to Norbsikck's version [12]. Some inodifications, concerning the start and the stepsize $h$, are introduced.

An automatic start calculates the high-order terms $a\left(x_{0}\right), b\left(x_{0}\right), c\left(x_{0}\right), d\left(x_{0}\right)$ and determines the initial stepsize $h_{0}$. The user is free in his choice for the start direction: backward/forward.

A backward start provides natural values for the lags in the high-order functions. However such a start is not always feasible, e.g. for differential equations with singularities backwards of $x_{0}$.

A forward start is applicable to all problems, but results in high-order functions running ahead with respect to the numerical integration, instead of lagging behind. 'The lags can be restored, using extrapolation (53) for the quantities $a, b, c$ and $d$, based on interval sizes $h$ and $h / 2$. These formulas are independent of the actual (unknown) values of the lags.

$$
a(x, h):=(64 \cdot a(x, h / 2)-17 \cdot a(x, h)) / 15, \quad b(x, h):=(64 \cdot b(x, h / 2)-9 \cdot b(x, h)) / 7,\}
$$

During the start large transients oceur when reversing the stepdirection.

Shifting the extraneous zero-positions is then less favorable, because of the loss of the finite $S^{4}$-oscillation reduction.

To avoid this difficulty our computer program uses NonDsIeck's original method for starting the integration, and one of our optimum methods for the actual solution of the differential equations. After starting the high-order function values can be adapted to their correct lagging values, corresponding to the extraneous zero-positions $z_{i}$ :

$$
\begin{aligned}
& a(x, h):=a(x, h)+\frac{-6+6 A-3 B+145 D}{1440 \cdot D} \cdot\left[f(x+h)-f^{[\mathrm{p}]}(x)\right] \\
& b(x, h):=b(x, h)+\frac{-2 A+3 B-2 C+100 D}{720 \cdot D} \cdot\left[f(x+h)-f^{[p]}(x)\right] \\
& c(x, h):=c(x, h)+\frac{-3 B+6 C+100 D}{1440 \cdot D} \cdot\left[f(x+h)-f^{[\mathrm{p}]}(x)\right] \\
& d(x, h):=d(x, h)+\frac{25 D-2 C}{1200 \cdot D} \cdot\left[f(x+h)-f^{[\mathrm{p}]}(x)\right] .
\end{aligned}
$$


The maximum stepsize is chosen, consistent with local requirements for stability and accuracy. Interval control consists of two tests, indicating whether or not $p$ lies inside the region of absolute stability and whether the global discretisation error is bounded by a prescribed accuracy parameter $\varepsilon$ (see NonDSIECK [12]).

We do not follow Nordsieck's restriction for the factor $\beta$

$$
h^{\prime}=\beta \cdot h, \quad \beta=1 / 2 \text { or } \beta=2
$$

when changing the stepsize. Any value of $\beta$ is allowed, to insure a flexible adjustment to the local properties of the differential equations.

\section{Numerical experiments}

NoRDSrEck's integration method has been applied to systems of differential equations on a DEC 10 digital computer, using double precision wordlength (LONG REAL) to reduce the effect of roundoff errors. Numerical results confirm the theoretical improvements in stability and accuracy for optimum extraneous zero-positions. The quality of a particular Nordsieck method is determined by the global discretisation error and the number of function evaluations, used for the complete integration (assumed proportional to the CPU-time).

Using a variable stepsize and having shifted the extraneous roots to optimum zero-positions may lead to a $20 \%$ decrease in the number of function evaluations. The interval control mechanism enlarges the stepsize $h$ for methods with smaller truncation coefficients $H$, resulting in approximately the same global discretisation error and a reduction in the number of function evaluations, compared with NoRDsIECK's original method.

For a fixed stepsize the number of function evaluations over the integration interval is constant. Optimum accurate zero-positions directly result in smaller discretisation errors for the numerical solution.

Example: Consider the initial value problem, consisting of the following 5 linear differential equations:

$$
\left\{\begin{array}{l}
y_{1}^{\prime}(x)=-y_{1}, \quad y_{i}(0)=1, \quad i=1,2,3,4,5 \\
y_{3}^{\prime}(x)=-y_{2}-y_{3} \\
y_{3}^{\prime}(x)=y_{2} \\
y_{4}^{\prime}(x)=-y_{4} \sqrt{3}+y_{5}, \\
y_{3}^{\prime}(x)=-y_{4}
\end{array}\right.
$$

with the constant eigenvalues of the Jacobian matrix equally distributed in the complex left-half $p$-plane:

$$
\begin{cases}\lambda_{1}=-1, & \\ \lambda_{2}=-\frac{1}{2}+\frac{1}{2} i \sqrt{3}, & \lambda_{3}=\bar{\lambda}_{2}, \\ \lambda_{4}=-\frac{1}{2} \sqrt{3}+\frac{1}{2} i, & \lambda_{5}=\bar{\lambda}_{4} .\end{cases}
$$

The exact solution of (56) can easily be computed from (57):

$$
\left\{\begin{array}{l}
y_{1}(x)=\mathrm{e}^{-x}, \\
y_{2}(x)=\mathrm{e}^{-x / 2}\left(\cos \frac{1}{2} x \sqrt{3}-\sqrt{3} \sin \frac{1}{2} x \sqrt{3}\right), \\
y_{3}(x)=\mathrm{e}^{-x / 2}\left(\cos \frac{1}{2} x \sqrt{3}+\sqrt{3} \sin \frac{1}{2} x \sqrt{3}\right), \\
y_{4}(x)=\mathrm{e}^{-x / 3 / 2}\left[\cos \frac{1}{2} x+(2-\sqrt{3}) \sin \frac{1}{2} x\right], \\
y_{5}(x)=\mathrm{e}^{-x \sqrt{3} / 2}\left[\cos \frac{1}{2} x-(2-\sqrt{3}) \sin \frac{1}{2} x\right] .
\end{array}\right.
$$

Using a backward start and a variable stepsize during numerical integration over the interval $[0,6]$, we compare NoRDsIECK's original scheme with our method (31) (optimum accuracy, while preserving stability).

$\eta_{\mathrm{av}}, \eta_{\max }$ : average and maximum (over $i$ ) of Euclidian norm of the discretisation error $\underline{y}\left(x_{i}\right), \underline{y}_{i}$.

FE: number of function evaluations $f(x, \underline{y})$.

$T_{\text {cpu }}$ : $\quad$ CPU - time in milliseconds.

$\varepsilon$ : $\quad$ input parameter for accuracy test (upper bound for global discretisation error).

NoRDSIECK's scheme: formula $(5 j$

\begin{tabular}{lllll}
\hline$\varepsilon$ & $\eta_{\text {av }}$ & $\eta_{\max }$ & FE & $T_{\text {cpu }}$ \\
\hline $10^{-5}$ & $1.83 \times 10^{-8}$ & $3.94 \times 10^{-8}$ & 100 & 1282 \\
$10^{-6}$ & $1.65 \times 10^{-9}$ & $3.60 \times 10^{-8}$ & 144 & 1758 \\
$10^{-7}$ & $1.51 \times 10^{-10}$ & $3.32 \times 10^{-10}$ & 208 & 2433 \\
$10^{-8}$ & $1.44 \times 10^{-11}$ & $3.39 \times 10^{-11}$ & 300 & 3461 \\
$10^{-8}$ & $1.38 \times 10^{-12}$ & $3.33 \times 10^{-12}$ & 438 & 4916 \\
$10^{-10}$ & $1.34 \times 10^{-13}$ & $3.42 \times 10^{-13}$ & 640 & 7021
\end{tabular}


Optimum method: formula (31)

\begin{tabular}{lllrl}
\hline$\varepsilon$ & $\eta_{\text {av }}$ & $\eta_{\text {max }}$ & FE & $T_{\text {cpu }}$ \\
\hline $10^{-5}$ & $1.30 \times 10^{-8}$ & $2.94 \times 10^{-8}$ & 94 & 1195 \\
$10^{-6}$ & $1.29 \times 10^{-9}$ & $3.18 \times 10^{-9}$ & 132 & 1595 \\
$10^{-7}$ & $1.20 \times 10^{-10}$ & $3.00 \times 10^{-10}$ & 188 & 2168 \\
$10^{-8}$ & $1.19 \times 10^{-11}$ & $3.21 \times 10^{-11}$ & 270 & 3105 \\
$10^{-9}$ & $1.15 \times 10^{-12}$ & $3.23 \times 10^{-12}$ & 390 & 4367 \\
$10^{-10}$ & $1.14 \times 10^{-13}$ & $3.37 \times 10^{-13}$ & 562 & 6105 \\
\hline
\end{tabular}

As predicted, the optimum method (3I) reduces the number of function evaluations and the CPU-time, while at the same time maintaining at least the same accuracy.

For a constant stepsize $h=0.04$ over the interval $[0,10]$ (250 steps), the optimum methods (31), (32), (33) result in global discretisation errors, proportional to the change in the coefficient $H$ of the truncation error.

\begin{tabular}{lllll}
\hline & $\eta_{\text {av }}$ & $\eta_{\text {max }}$ & $\eta_{\text {av }} /\left(\eta_{\text {av }}\right)$ Nond. & $H /(H)$ Nonv. \\
NoRDsIECK & $1.09 \times 10^{-6}$ & $1.19 \times 10^{-5}$ & 1.00 & 1.00 \\
Method (31) & $0.40 \times 10^{-6}$ & $0.43 \times 10^{-5}$ & 0.37 & 0.35 \\
Method (32) & $1.12 \times 10^{-6}$ & $1.21 \times 10^{-5}$ & 1.03 & 1.00 \\
Method (33) & $0.64 \times 10^{-6}$ & $0.70 \times 10^{-5}$ & 0.59 & 0.57 \\
\hline
\end{tabular}

\section{Concluding remarks}

Shifting the extraneous zero-positions in the complex $\lambda$-plane is an attractive way of improving the stability and accuracy of NoRDsifck's integration method. For large systems of differential equations with complicated righthand sides and long integration intervals, in particular, this technique saves a significant amount of function evaluations, and thus computer time, during numerical integration.

Compared with the approach taken by FeHLberg [3] and Hull [9], to obtain optimal methods, our method gives a better insight into the consequences of shifting the zero-positions of the extraneous roots. This method can easily be applied to the several implementations of NoRDSIECk's method known from literature, e.g. GEAR [4], KOHFELD [10].

A further development could be the construction of self-adapting methods (see HuLL [9]). These methods modify not only the stepsize $h$, but also the coefficient values $A, B, C$ and $D$ during the numerical integration, in order to better adapt to the local properties of the differential equations.

\section{References}

1 Brown, R. R.; Riley, J. D.; Bennett, M. M., Stability properties of Adams-Moulton type methods, Math. Comput. 19 (1965), $90-96$.

2 Collatz, L., The Numerical Treatment of Differential Equations, Springer Verlag, Berlin 1966.

3 Fehlberg, E., Numerically Stable Interpolation Formulas, with Favorable Error Propagation for First- and Second-Order Differential Equations, NASA Techn. Note D 599, 1961.

4 Gear, C. W., Numerical Initial Value Problems in Ordinary Differential Equations, Prentice Hall, New Jersey 1971.

5 GrigoriefF, R. D., Numerik Gewöhnlicher Differentialgleichungen. Band 1, 2, Teubner, Stuttgart 1977.

6 Hall, G.; WatT, J. M., Modern Numerical Methods for Ordinary Differential Equations, Clarendon Press, Oxford 1976.

7 Hearn, A. C., REDUCE 2 User's Manual, Univ. of Utah, Salt Lake City 1973.

8 Henrici, P., Discrete Variable Methods in Ordinary Differential Equations, John Wiley, New York 1962.

9 Hull, T. E.; NewberY, A. C. R., Corrector formulas for multi-step integration methods, J. Soc. Industr. Appl. Math. 10 (1962), $351-369$.

10 KонғеL, J. J.; Thомpson, G. T., A modification of Nordsieck's method, using an off-step point, J. of ACM 15 (1968), $390-401$.

11 Lewis, H. R.; Stovatt, E. J., Comments on a floating-point version of NorDSIECK's scheme for the numerical integration of differential equations, Math. Comput. 21 (1967), $157-161$.

12 Nordsieck, A., On numerical integration of ordinary differential equations, Math. Comput. 16 (1962), $22-49$.

13 Nordsieck, A., Automatic numerical integration of ordinary differential equations, Proc. Symp. Appl. Math. 15 (1963), 241 - 250.

14 Osborne, M. R., On Nordsieck's method for the numerical solution of ordinary differential equations, BIT 6 (1966), 51 -57.

15 Skeel, R. D.; JACKson, L. W., Consistency of Nordsieck methods, SIAM J. Num. 14 (1977), 910-924.

16 Sterter, H. J., Analysis of Discretisation Methods for Ordinary Differential Equations, Springer Verlag, Berlin 1973.

17 Traas, C. R., High-Order Numerical Integration of Second-Order Ordinary Differential Equations by Means of Nordsrecr's Method, National Aerospace Laboratory NLR, Amsterdam 1969.

18 Wesenan, J. A., Norbsimck's Method for the Solution of Ordinary Differential Equations, Rescona N.V., Amsterdam 1965.

Received Mai 5, 1982, corrected version February 14, 1983

Authors' addresses: Jr. R. H. J. GmeLiG, Dept. of Mathematics, University of Amsterdam, Roetersstraat 15, 1018 WB Amsterdam, The Netherlands; Dr. C. R. Trass, Dept. of Appl. Mathematics, Twente University of Technology, P. O. Box 217,7500 AE Enschede, The Netherlands 